\title{
AN OVERVIEW OF CRYPTOGRAPHIC LEDGER AUTOMATION: PILLARS, HISTORY, TYPES, USES \&FUTURE SCOPE
}

\author{
Harsha Rahwani \\ Undergraduate Engineering Scholar \\ School of Computer Science \&Information \\ Automation, \\ ANAND International College of Engineering, \\ Jaipur, India
}

Abstract- Cryptographic ledger, also known as Shared Register Automation, constructs the chronicle of any fichero asset inflexible \&seethrough through dissemination \&cryptographic hachering.

A common affinity for understanding cryptographic ledger automation is any type of document app. When a document is created \&then spread it among a whole lot of people, the document is spread rather in contrast to copy or budge. This generates a disseminate distribution bond that gives access to everyone for document on the same second. Nobody is banned to wait for changes from the other party, all changes at the dock are taken in that second, the alters are totally see-through.

Also, the cryptographic ledger is tad bit complex in contrast to other document making app, but the similarity is not appropriate thus it reflects 3 important points of automation:

- Fichero assets are disseminated rather in contrast to duplicate or transferred.

- The property is disseminating, allowing full instantaneous access.

- The see-through leader of the changes maintains the integrity of the document, creating trust in the property.

Cryptographic ledger is an actually promising \&timeless automation as it allows reduce crimes, prevent crime, \&bring scalability to many applications.

Cryptographic ledger has three main components: blocks, nodes \&miners.

\author{
Neeraj Prakash Shrivastava \\ Associate Professor \\ School of Computer Science \&Information \\ Automation, \\ ANAND International College of Engineering, \\ Jaipur, India
}

Keywords - Cryptographic ledger, History, Type, Use, Future Range.

\section{INTRODUCTION}

At its most essential level, cryptographic ledger is truly a bond of quads, but not in the best feeling of those words. At the point when we state the words "quad" \&"bond" in the new tragic, people though really talking about computerized specifics ("quad") throw in the preview specifics base ("bond").

"Quad" is based, cryptographic ledger is made of computerized snippets in specifics. Majorly, thre are 3 points:

Quad hoard specifics of transfer as the day, hour, \&cent measure that you buy at the latest from FlipKart.

Quad hoard specifics of who is making the transfer. A quad to provide you the best experience saves your name in the database. Instead of using the provided name, all the things you have searched for are stored \&then distinguished.

Quad hoard all major information from quads. As we all know each other by names, just like that the quads have named it a "hacher" which allows us to remain separated from quad. Hacheres are unknown \&digitally coded.

Cryptographic ledger comprises of three significant plans: quads, pivots \& diggers.

Quads

Each bond is included $\mathrm{n}$ no. quads \&each quad is made of 3 huge parts:

The data in the quad. 
- $\quad$ A 32-piece number is an easily overlooked detail. The easily overlooked detail just created basically after quad is made, \&that makes a quad head hacher.

- $\quad$ The hacher is to reliably start with zeroes. \&it is a total of 256-piece no. which is an assistant of quad.

When head quad of bond is made, a seemingly insignificant detail makes the cryptographic hacher. Furthermore, information set aside in quad saw as checked and reliably joined to the easily overlooked detail and hacher except for if it's digger.

\section{Diggers}

Diggers are used to make latest quads towards bond by methods for a plan known as tunneling.

In a cryptographic record every quad have an interesting seemingly insignificant detail and hacher, yet additionally references the hacher of the past quad in the bond, so mining a quad isn't fundamental, particularly on huge securities.

Excavators utilize wonderful programming to manage the amazingly shocking math issue of finding a seemingly insignificant detail that makes a perceived hacher. Since the easily overlooked detail is just 32 bits and the hacher is 256 , there are around four billion potential seemingly insignificant detail hacher mixes that must be mined before the correct one is found. Precisely when that occurs, excavators are said to have discovered the "amazing seemingly insignificant detail" \&their quad is added to the bond.

Uncovering an improvement to any quad prior in the bond requires re-mining the quad along the change, yet the aggregate of the obstructs that come after. This is the clarification it's unimaginably hard to control cryptographic record improvement. Consider it is as "success in math" since finding awe inspiring seemingly insignificant detail requires an epic extent of time and preparing power.

Right when a quad is reasonably mined, the change is perceived by the absolute of the turns on the structure $\&$ the excavator is remunerated financially.

\section{Pivots}

One of the most critical plans in cryptographic record advancement is dispersal. No one PC or affiliation can have the bond. Or maybe, it is a dispersed record by methods for the turns related along the bond. Turns can be such an electronic device that keeps up copies of the cryptographic record \&keeps the framework working. 0

Each turn has its own copy of the cryptographic record \&the framework ought to algorithmic-ally underwrite any as of late burrowed impede for the attach to be invigorated, trusted \&checked. Since cryptographic records are direct, every action in the record can be helpfully checked \&seen. Each part is given a novel alphanumeric ID number that shows their exchanges. Consolidating open particulars along a plan of overseeing rules enables the cryptographic record to keep up reliability \&makes trust among customers. Essentially, cryptographic records can be thought of as the flexibility of trust by methods for advancement.

\section{HISTORY}

Cryptographic record development has quite recently been in nearness for a modest quantity of the time that the web has, so taking everything into account, there are so far huge upgrades to come. Without a doubt, even now, in any case, masters have begun to seclude the chronicled scenery of cryptographic record into in any occasion three noteworthy stages.

\section{Stage 1: Bitcoin \& Cyber cash}

While the examinations that would go into the cryptographic record were turning around in programming building frameworks, it was the pseudonymous maker of Bitcoin, Satoshi Nakamoto, who spread out the cryptographic record as we probably am careful it in the white paper for BTC. Subsequently, cryptographic record progression started along the Bitcoin compose. While cryptographic record has since kept on observing use in a huge blend of different areas, in some sense it was orchestrated wonderfully for this mechanized capital and for pushing the goals of front-line capital record quantifies considerably more comprehensively.

In the most trustworthy stages, cryptographic record set up the crucial clarification of a mutual open record that fortifies a cryptographic capital arrange. Satoshi's concept of cryptographic record utilizes 1-megabyte (MB) quads of focal points on bitcoin exchanges. Quads are related together through a complex cryptographic attestation process, framing an unchanging bond. Truth be told, even in its soonest deceptions, cryptographic record advancement set up colossal amounts of the focal highlights of these structures, which remain today. Without a doubt, 


\section{International Journal of Engineering Applied Sciences and Technology, 2020 \\ Vol. 5, Issue 2, ISSN No. 2455-2143, Pages 182-187 \\ Published Online June 2020 in IJEAST (http://www.ijeast.com)}

bitcoin's cryptographic record remains overall unaltered from these soonest endeavors.

\section{Stage 2: Smart Contracts}

As time went on, organizers started to recognize that a cryptographic record could accomplish more rather than just record exchanges. Makers of ethereum, for example, had the probability that focal points \&trust understandings could besides profit by cryptographic record the board. In this way, ethereum addresses the second-age of the cryptographic record progression.

The basic progress achieved by ethereum was the event to skillful understandings. Usually, contracts in the standard business world are overseen between two separate parts, directly \&again along different substances supporting the oversight strategy. Sharp understandings are those that are automatic on a cryptographic record. They are authorized by an occasion like the downfall of a pass date or the accomplishment of a specific worth objective; in like way, the sharp understanding oversees itself, making modifications changing and along out the dedication of outside segments.

By and by, we may in any case seat the new limit of mind-blowing understandings. In this manner, regardless of whether we have genuinely proceeded ahead to the subsequent time of the movement of cryptographic record is scrappy.

\section{Stage 3: The Future}

One of the important issues facing cryptographic record is scaling. Bitcoin remains mourned in terms of professional career arranging times and container necking. Different new motorized capital record standards have attempted to reevaluate their cryptographic records so as to suit these issues, yet along fluctuating degrees of achievement. Later on, one of the most basic upgrades getting ready for cryptographic record improvement continuing will probably need to do along adaptability.

Thus, new vocations of cryptographic record progression are being found \& executed ceaselessly. It's hard to state unequivocally where these degrees of progress will lead the headway \& the cryptographic capital industry all things considered. Supporters of cryptographic record are likely going to discover this incredibly enabling; from their viewpoint, we are living in a second along an epochal progression that is proceeding to make and spread out.

\section{TYPES OF CRYPTOGRAPHIC LEDGER}

There are three essential sorts of cryptographic ledgers, which do exclude conventional specifics bases or dispersed record innovation (DLT) that are regularly mistaken for cryptographic ledgers.

\section{Public cryptographic ledgers like Bitcoin \&Ethereum}

- Public cryptographic records are planned to be totally spread, along no one individual or component controlling which trades are recorded in the cryptographic record or the solicitation wherein they are readied.

- Public cryptographic records can be significantly control safe, since anyone is accessible to join the framework, paying little regard to territory, nationality, \&so on. This makes it incredibly hard for experts to shut them down.

- Lastly, open cryptographic records all have a token related along them that is consistently proposed to help \&prize individuals in the framework.

\section{Private cryptographic ledgers like Hyperledger \&R3 Corda}

Such a bonds are private cryptographic records, regardless called apportioned cryptographic records, have diverse perceptible complexities from open cryptographic records.

- Participants need agree to join the structures

- Transactions are private \&are just open to natural structure people that have been allowed to join the system

- Private cryptographic records are continuously intertwined rather than open cryptographic records.

Private cryptographic records are gigantic for attempts who need to coordinate and offer information, yet needn't mess with their touchy business information obvious on an open cryptographic record. These bonds, by their tendency, are dynamically joined together; the substances running the bond have huge comm\&over people \&administration structures. 


\section{International Journal of Engineering Applied Sciences and Technology, 2020 \\ Vol. 5, Issue 2, ISSN No. 2455-2143, Pages 182-187 \\ Published Online June 2020 in IJEAST (http://www.ijeast.com)}

Private cryptographic records might have a token associated with along the bond.

\section{Hybrid cryptographic ledgers like Dragonbond}

Dragonbond has a unique spot inside the cryptographic record natural framework in that it's a cross variety cryptographic record. This suggests it merges the insurance favorable circumstances of a permissioned \& private cryptographic record along the security \&straightforwardness points of interest of an open cryptographic record. That gives associations critical flexibility to pick what data they have to make open \&straightforward \&what data they have to keep covered up.

- The hybrid nature of Mythical beast bond cryptographic record stage is made possible by our ensured Interbond ${ }^{\mathrm{TM}}$ capacity, which licenses us to helpfully relate along other cryptographic record shows. Considering a multi-bond arrangement of cryptographic records

- This handiness makes it essential for associations to work along the straightforwardness they are scanning for, along out surrendering security \&protection.

- Also, having the choice to post to various open cryptographic records promptly fabricates the security of trades, as they benefit by the joined hacher power being applied to the open bonds.

\section{3 PILLARS OF CRYPTOGRAPHIC LEDGER}

The three primary properties of Cryptographic ledger Automation which have helped it increase across the board approval are as per the following:

- Dissemination

- See-through

- Permanence

\section{Column 1: Dissemination}

Before Bitcoin \&BitTorrent came, we were progressively used to blend organizations. The idea is very essential. You have a united substance that set aside all the data \&you'd need to work together only along this component to get whatever points of interest you required.
Another instance of a concentrated structure is the banks. They crowd all your lawful delicate, \&the fundamental way that you can pay someone is by encountering the bank.

The regular client server model is an arrangement 1 model.

By and by, blend systems have compensated us well for quite a while, not a long standing, they have a couple of vulnerabilities.

Directly off the bat, since they are united, all the data is taken care of in one spot. This makes them evident target spots for likely developers.

In case the united structure were to encounter an item overhaul, it would stop the entire system

Think about how conceivable it is that the united component somehow shuts down for no good reason. That way nobody will have the alternative to get to the points of interest that it has.Most critical result comprehensible, think about how conceivable it is that this component gets spoiled \&malevolent. In case that happens, by then all the data that is inside the cryptographic record will be sabotaged.

Anyway, what happens if we just evacuate this united substance?

In a spread system, the points of interest aren't taken care of by one single substance. Believe it or not, every person in the framework asserts the points of interest.

In a disperse framework, in case you expected to interface along your friend, by then you can do so really along out encountering an untouchable. That was the essential conviction framework behind Bitcoins. You \&just just you are answerable for your legitimate delicate. You can send your legitimate delicate to anyone you need along out encountering a bank.

\section{Column 2: See-through}

Only thing fascinating \& misinterpreting plans in cryptographic ledger is "candor." A few people say that cryptographic ledger allows the certainty while some proclaim that it is certain. Why do you think that happens?

A person's personality is lined up via a hard algorithm $\&$ tell just via their open access point. Along these lines, even if you somehow see the transfer if money, you won't see "I have one coin" rather you will see "1MF1bhsFLkBzzz9vpFYEmyCt7NZJ one BTL". 


\section{International Journal of Engineering Applied Sciences and Technology, 2020 Vol. 5, Issue 2, ISSN No. 2455-2143, Pages 182-187 \\ Published Online June 2020 in IJEAST (http://www.ijeast.com)}

Thusly, while the person's certified character is secure, you will at present watch all the trades that were done by their open area. This level of straightforwardness has never lived inside a capital record structure. It incorporates that extra, \& truly vital, level of obligation which is required by a part of these most noteworthy establishments.

Talking completely from the viewpoint of record cash, if you know the open area of one of these immense associations, you can simply pop it in a traveler \& take a gander at all the trades that they have involved along. This forces them to be completely blunt, something that they have never expected to oversee.

\section{Column 3: Permanence}

Permanence, along with the cryptographic ledger, tells that if anything goes through the cryptographic ledger, it cannot be altered.

Can you think how important it will be for asset foundations?

Think how much of theft cases will stop to occur and really rising only if the people will realize not to mess with company's assets.

The intention as to why cryptographic ledger gets this scheme is because of that ledger hacher work.

Basically, hachering means to pull any particular length of input string and then fixing its length. Along with cyber cash like bitcoin, transfer is done as info \& passes through a hachering algorithm.

\section{USES OF CRYPTOGRAPHIC LEDGER}

- Asset The executives: Exchange Handling \&Settlement.

- Insurance: Cases handling.

- Payments: Cross-Outskirt Instalments.

- Unconventional legal tender loan specialists/hard legal tender loaning.

- Your vehicle/cell phone.

- Cryptographic ledger Web of-Things (IoT)

- Smart Machines.

- Supply Bond Sensors.

\section{FUTURE SCOPE}

Cryptographic ledger innovation has an extraordinary future around the world. An extraordinary extent of Cryptographic ledger innovation has been seen in the capitalledger field. The budgetary associations couldn't adequately deal along the substantial outstanding burden after demonetization \&in this way drawn out the issues of having a concentrated master for taking care of the capitalledger exchanges. Subsequently, the RBI is moving banks to support digitization. They have likewise discharged an explanation which underscored the likelihood of Cryptographic ledger to battle faking \&the odds of achieving specific adjustments in the working of capital related markets, insurance distinguishing proof \&installment framework. Consolidating Cryptographic ledger along budgetary exchanges gives out astounding advantages, for example, a lot of time \&legal tender could be spared, remembering an exceptional decrease for time required for preparing \&approving exchanges. The cryptographic ledger capacities on a conveyed specifics base which make the tasks easily, guaranteeing tight security, \&made it safe from fichero assaults.

In the wake of perceiving the advantages of Cryptographic ledger Innovation, a few budgetary organizations have begun spending impressively in this specific field. Cryptographic ledger can likewise help in shortening the progression of dark legal tender \&managing the broad legal tender cleaning in the economy in light of the fact that each address utilized for exchanges is put away everlastingly on the specifics bases, making all the exchanges provable \&dependable. The administration is watching Cryptographic ledger as an approach to investigate a scope of alternatives which may assist along applying a fitter control on the country's economy.

Cryptographic ledger Innovation is one of the most reliable advances when it requires to monitor capital related properties. Cryptographic ledger innovation has pulled in numerous organizations who need to include the unmistakable highlights of it to their security structures. Numerous examinations have been done for computerized capital ledger standards \&cryptographic ledger innovation, which speaks to that both of these advances will be proceeding to upset the world.

Aside from capital related enterprises, cryptographic ledger innovation additionally has a splendid future in different parts.

\section{CONCLUSION}

The utilization of Cryptographic ledger innovation isn't restricted uniquely to the account business. It has a phenomenal future in various areas, for example, gracefully bond the board, computerized publicizing, determining, ficherosecurity, Web of things, organizing, \&so on. Cryptographic ledger innovation 


\section{International Journal of Engineering Applied Sciences and Technology, 2020 \\ Vol. 5, Issue 2, ISSN No. 2455-2143, Pages 182-187 \\ Published Online June 2020 in IJEAST (http://www.ijeast.com)}

additionally has an enormous imminent to give the new openings to occupation in the business. It likewise improves the expert's ability to update themselves. Along the assistance of Cryptographic ledger innovation, it is conceivable to change the entire world into a lot littler spot. The value-based exercises can be performed a lot quicker \&proficiently utilizing Cryptographic ledger. Cryptographic ledger innovation will be utilized in a lot more areas later on, for example, in government frameworks as these frameworks are moderate, thick, \&liable to defilement. Executing Cryptographic ledger innovation in government framework can make their activities considerably more secure \&proficient.

\section{REFERENCES}

[1] "State of cryptographic ledger q1 2016: Cryptographic ledger funding over takesbitcoin," 2016. [Online]. Available: http://www.coindesk.com/state-of- cryptographic ledger-q1- 2016/

[2] S. Nakamoto, "Bitcoin: A peer-to-peer electronic legal tender system," 2008.[Online]. Available: https://bitcoin.org/bitcoin.pdf

[3] G. W. Peters, E. Panayi, \&A. Chapelle, "Trends in crypto-currencies\&cryptographic ledger technologies: A capitalledger theory \&regulationperspective," 2015. [Online]. Available: http://dx.doi.org/10.2139/ssrn.2646618

[4] G. Foroglou \&A.-L. Tsilidou, "Further applications of the cryptographic ledger,"

2015.

[5] A. Kosba, A. Miller, E. Shi, Z. Wen, \&C. Papamanthou, "Hawk:

The cryptographic ledger model of cryptography \&privacy-preserving smart

contracts," in Proceedings of IEEE Symposium on Security \&Privacy

(SP), San Jose, CA, USA, 2016, pp. 839-858

[6]. Back, "Hacherlegal tender - a denial of service countermeasure," http://www.hacherlegal tender.org/papers/hacherlegal tender.pdf, 2002.

[7]. Hawk: The Cryptographic ledger Model of Cryptography \&Privacy-Preserving Smart Contracts. / Kosba, Ahmed; Miller, Andrew; Shi, Elaine; Wen, Zikai; Papamanthou, Charalampos.
[8]. NRI: Survey on cryptographic ledger technologies \&related services. Tech. rep. (2015).

[9]. Moindrot, O. (2017). Proof of Stake Made Simple along Casper.

[10].L. Lamport, R. Shostak, \&M. Pease, "The byzantine generals problem," ACM Transactions on Programming Languages \&Systems (TOPLAS), vol. 4, no. 3, pp. 382-401, 1982.

[11] C. K. Frantz \&M. Nowostawski, "From institutions to code: Towards automated generation of smart contracts," in 2016 IEEE 1st International Workshops on Foundations \&Applications of Self* Systems (FAS*W), pp. 210-215, IEEE, 2016.

[12] B. Marino \&A. Juels, "Setting standards for altering \&undoing smart contracts," in International Symposium on Rules \&Rule Markup Languages for the Semantic Web, pp. 151-166, Springer, 2016.

[13] T. Chen, X. Li, X. Luo, \&X. Zhang, "Underoptimized smart contracts devour your money," in 2017 IEEE 24th International Conference on Software Analysis, Evolution \&Reengineering (SANER), pp. 442-446, IEEE, 2017.

[14] F. Idelberger, G. Governatori, R. Riveret, \&G. Sartor, "Evaluation of logic-based smart contracts for cryptographic ledger systems," in International Symposium on Rules \&Rule Markup Languages for the Semantic Web,167-183, Springer, 2016.

Neeraj Prakash Shrivastava Professor \&Head of School at School of Computer Science Engineering \&IT - An\&International College of Engineering, Jaipur, Rajasin contrast to, India.

He is having 21+ Yrs. Experience in Academia.

Harsha Rahwani is undergraduate scholar pursuing her final semester of engineering in computer science from An\&International College of Engineering, Jaipur, which is affiliated to Rajasin contrast to Technical University, Kota, Rajasin contrast to, India. 